\title{
EPIDEMIOLOGY AND ANTIBIOTIC SENSITIVITY OF STAPHYLOCOCCUS AUREUS NASAL CARRIAGE IN CHILDREN IN HUNGARY
}

\author{
Krisztina LaUb, AdRIENN TÓthPÁL, SZILVIA Kardos \\ and ORSOLYA DOBAY* \\ Institute of Medical Microbiology, Semmelweis University, Budapest, Hungary
}

(Received: 1 September 2016; accepted: 5 December 2016)

\begin{abstract}
The aim of this study was to assess the Staphylococcus aureus nasal carriage rate in healthy children all over Hungary and to specify some risk factors, the antibiotic resistance patterns of the bacteria, and their genetic relatedness. In total, 878 children (aged 3-6 years) were screened at 21 day-care centers in 16 different cities in Hungary, between February 2009 and December 2011. Samples taken from both nostrils were cultured on blood agar, and suspected $S$. aureus isolates were identified by $\beta$-hemolysis, catalase positivity, clump test, and nucA PCR. Methicillin-resistant strains were screened by mecA and mecC PCR. Antibiotic susceptibility was determined by agar dilution or gradient test strips. Pulsed-field gel electrophoresis was used for genotyping. S. aureus carriage rate was found to be $21.3 \%$, which correlates well with international data. We found no statistically significant correlation between the gender or the sibling status and $S$. aureus carriage. All isolates were sensitive to oxacillin, trimethoprim-sulfamethoxazole, and mupirocin. The resistance rates for erythromycin, ciprofloxacin, clindamycin, gentamicin, and tetracycline were $7.5 \%$, $0.5 \%, 1.1 \%, 3.7 \%$, and $4.3 \%$, respectively. The isolates showed very high genetic diversity. In summary, carried $S$. aureus isolates are more sensitive to antibiotics compared with clinical isolates in Hungary, and methicillin-resistant $S$. aureus carriage rate is very low yet.
\end{abstract} CA-MRSA

Keywords: Staphylococcus aureus, nasal carriage, antibiotic resistance,

\section{Introduction}

Staphylococcus aureus ( $S$. aureus) is one of the most important pathogens for humans and continues to be one of the most important nosocomial agents also. It colonizes the skin and mucosal surfaces, and the most consistent

\footnotetext{
*Corresponding author; E-mail: dobay.orsolya@med.semmelweis-univ.hu
} 
site from which this bacterium can be cultured is the anterior nares [1]. Children attending communities are well-known carriers of several pathogenic bacteria, such as $S$. aureus. As nasopharyngeal colonization of these bacteria can lead to the development of severe diseases - such as skin and soft tissue infections, pneumonia, bloodstream infections, and bone and joint infections [2] - in the carrier itself or in susceptible contacts (e.g., grandparents or immunocompromised persons), it is very important to explore the carriage rate.

The typical S. aureus carriage rate is $10 \%-35 \%$ in children and approximately $35 \%$ in the general adult population [3]. Ghasemzadeh-Moghaddam et al. [4] demonstrated in adults that $S$. aureus nasal carriage is a determinant for acquiring new $S$. aureus strains. They also have shown that the nasal carriage predisposes to nosocomial acquisition of new strains during hospitalization. The airborne transmission of $S$. aureus is affected by many factors, for example, the number of bacteria colonizing the nares. There are several papers about outbreaks due to airborne dispersal of $S$. aureus in association with skin colonization or viral upper respiratory tract infection of either health-care workers or patients, which is called the "cloud" phenomenon [5]. This phenomenon was established in the case of newborns and adults as well. As much as $75 \%$ of newborns carrying $S$. aureus dispersed this bacterium from their noses into the air once they acquired an upper respiratory tract infection [6]. Young children might play a special role in horizontal spreading of bacteria as their nasopharynx shows some special features, such as immature immunological response and high susceptibility to viral respiratory infections [7].

Penicillin-resistant $S$. aureus strains have arisen quickly after the introduction of this first drug, but an even greater concern was the emergence of methicillin resistance in 1961. These first methicillin-resistant S. aureus (MRSA) clones have soon been spreading worldwide, but first only in the hospital setting. In the USA, MRSA is still ranking high in the list of microbes causing lethal infections [8]. In the recent years, however, especially the emergence of community-acquired methicillin-resistant $S$. aureus (CA-MRSA) strains is an alarming problem, possessing increased virulence and spreading abilities compared with the hospital-acquired methicillin-resis$\operatorname{tant} S$. aureus isolates [9]. This has increased the importance of screening and monitoring $S$. aureus circulation in the community and its antibiotic susceptibility as well [10].

The aim of this study was to assess the $S$. aureus carriage rate in young children all over Hungary and to specify some risk factors, the bacteria's antibiotic resistance patterns, and their genetic relatedness. 


\section{Materials and Methods}

\section{Study population}

In total, 878 healthy children (aged 3-6 years) were screened at 21 day-care centers (DCCs) in 16 different cities in Hungary, between February 2009 and December 2011. Before the sample collection, parents were provided by a questionnaire in which they agreed to the process and provided some data. In 35 cases, in the absence of appropriate data, the gender of children was unknown. In the remaining 843 cases, genders were represented almost equally: $50.9 \%$ $(n=429)$ males and $49.1 \%(n=414)$ females. For statistical analysis, where appropriate, the $\chi^{2}$ test was used.

\section{Sample collection}

Samples were taken from both nostrils with soft cotton swabs and inserted into active charcoal containing Amies transport media (Transwab, Medical Wire \& Equipment, Corsham, UK). The swabs were transported to the microbiology laboratory within $24 \mathrm{~h}$.

\section{Phenotypical identification}

The samples were inoculated onto Mueller-Hinton blood agar plates, and after an overnight incubation at $37^{\circ} \mathrm{C}$, in $5 \% \mathrm{CO}_{2}$, the $\beta$-hemolytic colonies were isolated to produce pure culture. The identity of $S$. aureus isolates was confirmed by colony morphology, catalase test, and clump test (Pastorex Staph-Plus Kit, Bio-Rad, Marnes-la-Coquette, France). The clump-positive isolates were frozen and kept at $-80^{\circ} \mathrm{C}$ on cryobeads (Cryobank, Mast Diagnostica, Bootle, UK) until further examinations.

\section{Genotypical identification}

To confirm the identity of the isolates at the genetic level, nucA PCR was also performed to detect the thermonuclease gene, which is species-specific for S. aureus. MRSA screening was done by PCR to detect mecA or mecC genes. $n u c \mathrm{~A}$ and mecA amplifications were performed in a multiplex PCR reaction, where both primer pairs were designed in our laboratory with the Primer3 program [11]: 
nucA forward: 5'-ATGGACGTGGCTTAGCGTAT-3'

nucA reverse: 5'-TGACCTGAATCAGCGTTGTC-3'

$\mathrm{PCR}$ product $=193 \mathrm{bp}$

mecA forward: 5'-CCCAATTTGTCTGCCAGTTT-3'

mecA reverse: 5'-ATCTTGGGGTGGTTACAACG-3'

$\mathrm{PCR}$ product $=538 \mathrm{bp}$

Briefly, two and a half microliter of boiled bacterium templates $\left(99^{\circ} \mathrm{C}\right.$, $15 \mathrm{~min}$ ) were used in the PCR reaction, with the following cycling parameters: 3 min at $94{ }^{\circ} \mathrm{C}$, then 30 cycles of $94{ }^{\circ} \mathrm{C} 60 \mathrm{~s}, 54{ }^{\circ} \mathrm{C} 60 \mathrm{~s}$, and $72{ }^{\circ} \mathrm{C} 30 \mathrm{~s}$, and a final extension of $72^{\circ} \mathrm{C}$ for $10 \mathrm{~min}$. The PCR always included ATCC 33591 as a nucA, mecA positive external control.

$m e c \mathrm{C}$ PCR was done in a separate reaction as described by Paterson et al. [12], and the ATCC BAA-2312 strain was used as mecC positive control.

\section{Antibiotic susceptibility testing}

The minimum inhibitory concentration (MIC) to penicillin, oxacillin, erythromycin, clindamycin, tetracycline, gentamicin, ciprofloxacin, and mupirocin was determined by agar dilution in Mueller-Hinton plates, using an A400 Multipoint Inoculator (AQS Manufacturing Ltd., Southwater, UK). Penicillin susceptibility was also checked with 1UI disks (Bio-Rad), because disk diffusion is more reliable than MIC determination for the detection of penicillinase producer $S$. aureus isolates as suggested by the European Committee on Antimicrobial Susceptibility Testing (EUCAST) [13]. For phenotypic screening of MRSA, cefoxitin disks (30 $\mu \mathrm{g}$, Bio-Rad) were used in addition to oxacillin MICs [13]. In the case of trimethoprimsulfamethoxazole (TMP/SMX), we used gradient test strips (Liofilchem, Roseto degli Abruzzi, Italy). In all cases, the EUCAST guidelines and breakpoints were applied [13]. ATCC 29213 was used as control strain.

For the isolates resistant to erythromycin but sensitive to clindamycin, double disk diffusion was performed to detect inducible clindamycin resistance, using $15 \mu \mathrm{g}$ erythromycin and $2 \mu \mathrm{g}$ clindamycin disks (Bio-Rad).

\section{Genotyping by pulsed-field gel electrophoresis}

To determine the genetic relatedness of the isolates, pulsed-field gel electrophoresis (PFGE) was used as described earlier [14]. The complete bacterial genome was embedded in agarose plugs and lysed in several steps to purify DNA. 
For the digestion, Sma I restriction enzyme was used $\left(3 \mathrm{~h}, 25^{\circ} \mathrm{C}\right)$. The digested samples were run in a $1 \%$ agarose gel along with N0340S Lambda Ladder PFG Marker (New England Biolabs, Hitchin, Hertfordshire, UK), in a Bio-Rad CHEF$\mathrm{DR}^{\circledR}$ II PFGE machine, for $21 \mathrm{~h}$ at $14{ }^{\circ} \mathrm{C}$, with the following pulse times: block one $-5 \mathrm{~s} / 15 \mathrm{~s}$ for $10 \mathrm{~h}$, and block two $-15 \mathrm{~s} / 60 \mathrm{~s}$ for $11 \mathrm{~h}$. After the gel image was captured, the PFGE patterns were analyzed and the dendrograms were created by the Fingerprinting II software (Bio-Rad).

\section{Results}

\section{Carriage rate and risk factors}

Among the 878 children, 187 carried $S$. aureus which equals to a $21.3 \%$ carriage rate. Analyzing the questionnaires filled in by the parents, the gender of the carrier children was known in 179 out of the 187 cases: there were $96(53.6 \%)$ males and 83 (46.4\%) females. Another piece of information about the children was whether they had siblings or not. Among the carriers with known sibling status $(n=177)$, slightly higher percentage had siblings $(n=139,78.5 \%)$, compared with the non-carriers $(n=655)$, where $73.6 \%(n=482)$ had siblings. However, we found no statistically significant correlation between any of these potential risk factors and $S$. aureus carriage as shown in Table I.

\section{Antibiotic susceptibility}

Most of the isolates $(92.0 \%, n=172)$ were resistant to penicillin (Table II). Interestingly, there was one strain that had an MIC of $0.064 \mathrm{mg} / \mathrm{L}$ but it was resistant based on the disk diffusion test, so it was considered resistant according the EUCAST guidelines. However, all the penicillin-resistant isolates were susceptible to oxacillin and neither the mecA nor the mec $\mathrm{C}$ gene could be detected. The isolates with the highest oxacillin MICs $(0.5$ or $1 \mathrm{mg} / \mathrm{L})$ were

Table I. Correlation between the analyzed risk factors and Staphylococcus aureus carriage

\begin{tabular}{lccc}
\hline Risk factors & S. aureus carriers & S. aureus non-carriers & $p$-value $\left(\chi^{2}\right.$ test $)$ \\
\hline Male & $96(22.4 \%)$ & $333(77.6 \%)$ & NS $(p=0.41)$ \\
Female & $83(20.0 \%)$ & $331(80.0 \%)$ & NS $(p=0.18)$ \\
Having siblings & $139(22.4 \%)$ & $482(77.6 \%)$ & \\
Not having siblings & $38(18.0 \%)$ & $173(82.0 \%)$ & \\
\hline
\end{tabular}

Note: NS: not significant. 
Table II. Antibiotic susceptibility pattern of the carried Staphylococcus aureus isolates $(n=187)$

\begin{tabular}{lclllll}
\hline Antibiotic & $\begin{array}{c}\text { MIC range } \\
{[\mathrm{mg} / \mathrm{L}]}\end{array}$ & $\begin{array}{c}\mathrm{MIC}_{50} \\
{[\mathrm{mg} / \mathrm{L}]}\end{array}$ & $\begin{array}{l}\text { MIC } 90 \\
{[\mathrm{mg} / \mathrm{L}]}\end{array}$ & $\begin{array}{c}\text { EUCAST } \\
\text { breakpoint } \\
{[\mathrm{mg} / \mathrm{L}]}\end{array}$ & $\begin{array}{c}\text { Sensitive } \\
\%(n)\end{array}$ & $\begin{array}{c}\text { Resistant } \\
\%(n)\end{array}$ \\
\hline Penicillin & 0.032 to 128 & 4 & 16 & $\mathrm{~S} \leq 0.125$, & $8.6 \%(16)$ & $92.0 \%(172)$ \\
& & & & $\mathrm{R}>0.125$ & & \\
Oxacillin & 0.125 to 1 & 0.25 & 0.5 & $\mathrm{~S} \leq 2, \mathrm{R}>2$ & $100 \%(187)$ & $0.0 \%(0)$ \\
TMP/SMX & 0.008 to 0.25 & 0.023 & 0.032 & $\mathrm{~S} \leq 2, \mathrm{R}>4$ & $100 \%(187)$ & $0.0 \%(0)$ \\
Mupirocin & $\leq 0.032$ to 0.125 & 0.064 & 0.125 & $\mathrm{~S} \leq 1, \mathrm{R}>256$ & $100 \%(187)$ & $0.0 \%(0)$ \\
Ciprofloxacin & 0.125 to $>1$ & 0.5 & 1 & $\mathrm{~S} \leq 1, \mathrm{R}>1$ & $99.5 \%(186)$ & $0.5 \%(1)$ \\
Erythromycin & 0.25 to $>512$ & 0.5 & 0.5 & $\mathrm{~S} \leq 1, \mathrm{R}>2$ & $92.5 \%(173)$ & $7.5 \%(14)$ \\
Clindamycin & 0.064 to $>512$ & 0.125 & 0.125 & $\mathrm{~S} \leq 0.25, \mathrm{R}>0.5$ & $98.9 \%(185)^{\mathrm{a}}$ & $1.1 \%(2)$ \\
Gentamicin & 0.125 to 2 & 1 & 1 & $\mathrm{~S} \leq 1, \mathrm{R}>1$ & $96.3 \%(180)$ & $3.7 \%(7)$ \\
Tetracycline & $\leq 0.064$ to 64 & 0.25 & 0.5 & $\mathrm{~S} \leq 1, \mathrm{R}>2$ & $95.7 \%(179)$ & $4.3 \%(8)$ \\
\hline
\end{tabular}

${ }^{\mathrm{a}}$ Eleven isolates out of these had inducible resistance.

tested also with $30 \mu \mathrm{g}$ cefoxitin disks, and all showed sensitivity (26-27 mm inhibition zones), so there were no discrepancies between the oxacillin and cefoxitin results. All isolates were sensitive to TMP/SMX and mupirocin, while the highest resistance was observed in the case of erythromycin $(7.5 \%)$. The resistance rates for ciprofloxacin, clindamycin, gentamicin, and tetracycline were $0.5 \%, 1.1 \%, 3.7 \%$, and $4.3 \%$, respectively. There were 11 isolates expressing high-level resistance to erythromycin ( $\mathrm{MIC} \geq 512 \mathrm{mg} / \mathrm{L}$ ) but phenotypically sensitive to clindamycin $(\mathrm{MIC}=0.125 \mathrm{mg} / \mathrm{L})$, all of these had inducible clindamycin resistance based on the double disk diffusion test.

\section{Genetic relatedness}

The PFGE analysis was performed in the case of 59 strains. Based on the PFGE patterns, the isolates showed very high diversity (Figure 1); however, three major clusters could be detected. Even within the same DCC group, the PFGE patterns differ by several bands (see, e.g., the upper lanes). On the other hand, sometimes the very same clone could be found in two distant cities (e.g., Györ in NW Hungary and Debrecen in NE Hungary).

\section{Discussion}

In this study, the occurrence of $S$. aureus nasal carriage of healthy 3-6-year-old children in Hungary was found to be $21.3 \%$, which correlates well with international data $[3,15]$. A cross-sectional study published in 2013 compared the prevalence 


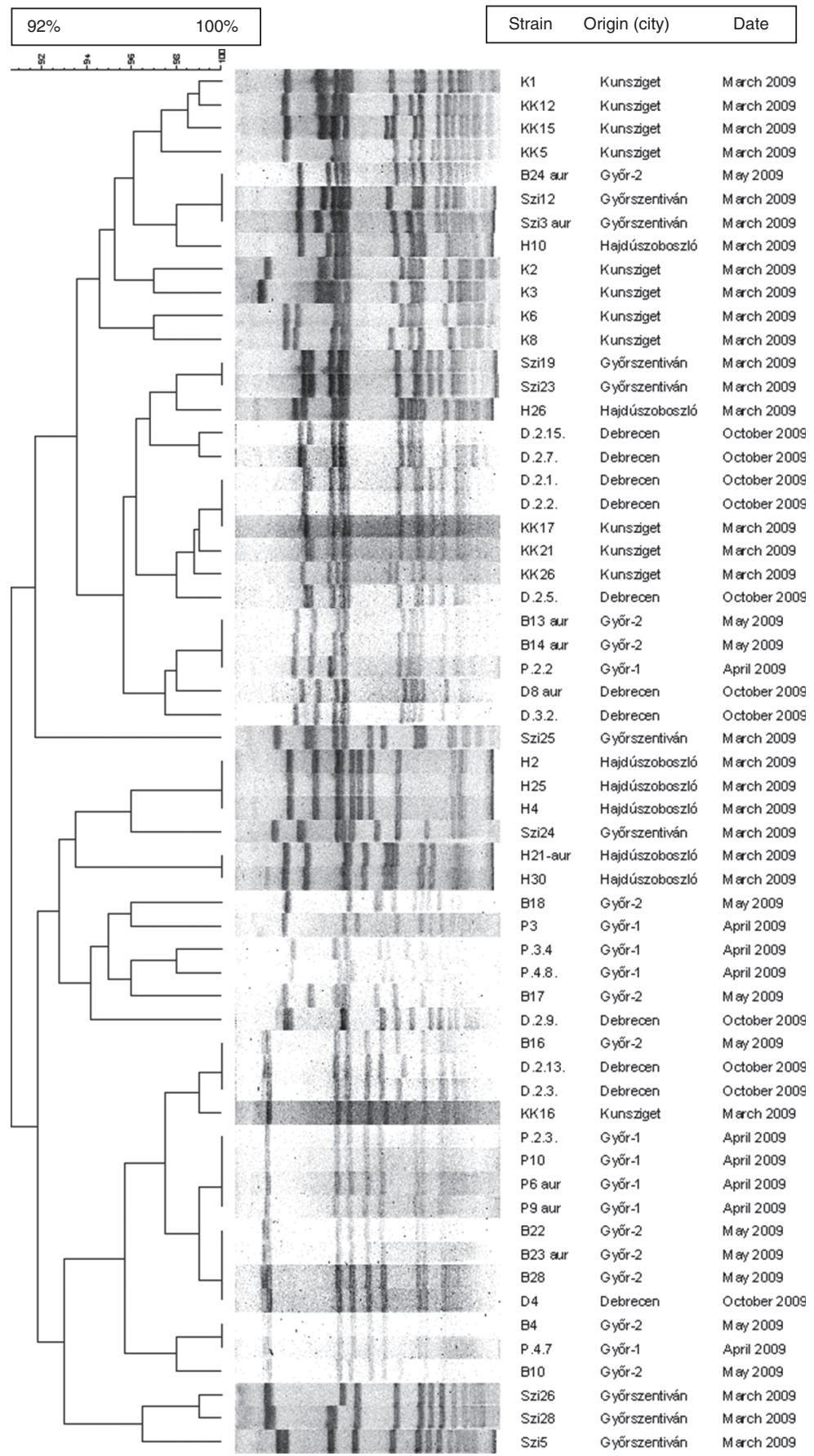

Figure 1. Pulsed-field gel electrophoresis dendrogram of 59 Staphylococcus aureus strains (2009-2011) 
of nasal $S$. aureus carriage of healthy patients in nine European countries (involving $>32,000$ people), including Hungary [16]. The overall carriage rate $(21.6 \%)$ almost equals to the average carriage rate found by us. However, this study included patients of all ages. In the age category of 4-19 years, the lowest prevalence was found in Hungary (19.9\%, out of 1,133 patients tested), whereas the highest $(36.2 \%)$ was seen in The Netherlands. Nonetheless, there are even higher differences in carriage rates, for instance, in an Indian study, the prevalence of $S$. aureus nasal colonization was found to be $52.3 \%$ in 5-15-year-old children [17]. Another study was conducted in Kayseri, Turkey, where $18 \%$ of healthy preschool children (aged 0-6 years) were colonized by S. aureus [18].

This $21.3 \%$ carriage rate is lower than was previously found by us among healthy young adults, namely third-year university students in Hungary [11], where both Hungarian as well as international students about 21 years of age were screened and $29.3 \%$ of them proved to be carriers.

Although the male-female ratio of the screened children in this study was quite equalized $(50.9 \%$ versus $49.1 \%$ ), males slightly (but not statistically significantly) dominated among carriers (53.6\% versus $46.4 \%)$. Wertheim et al. [19] stated in a review that $S$. aureus carriage rate is higher in men. In the abovementioned paper of den Heijer et al. [16], a similar conclusion was established, specifically that men had a higher chance for nasal carriage than did women. On the other hand, however, there are several papers where there is no correlation between gender and carriage [9, 10, 20, 21].

Similarly to gender, having siblings in the family was not shown to be statistically significant either as a possible risk factor in this study. Esposito et al. [10] demonstrated similar conclusion in an Italian study, in the case of school children.

It can be established that carried $S$. aureus isolates are more sensitive to many antibiotics compared with clinical isolates in Hungary. National annual antibiotic sensitivity data are available from the National Center for Epidemiology (NCE), separated to inpatients and outpatients. We looked at the same time period (i.e., 2009-2011), including data of 13,000-23,000 people yearly [22]. Naturally, inpatients data show higher resistance than outpatients data, especially for oxacillin and ciprofloxacin (MRSA in hospitals), but the resistance rates of the carried isolates from this study are even lower (except for penicillin and gentamicin). These differences are shown in Figure 2.

A very important question is MRSA rate. According to the NCE data, oxacillin resistance was $7.2 \%$ in outpatients and $23.5 \%$ in inpatients in 2011 [22]. MRSA among outpatients has been showing a slow but continuous increase 


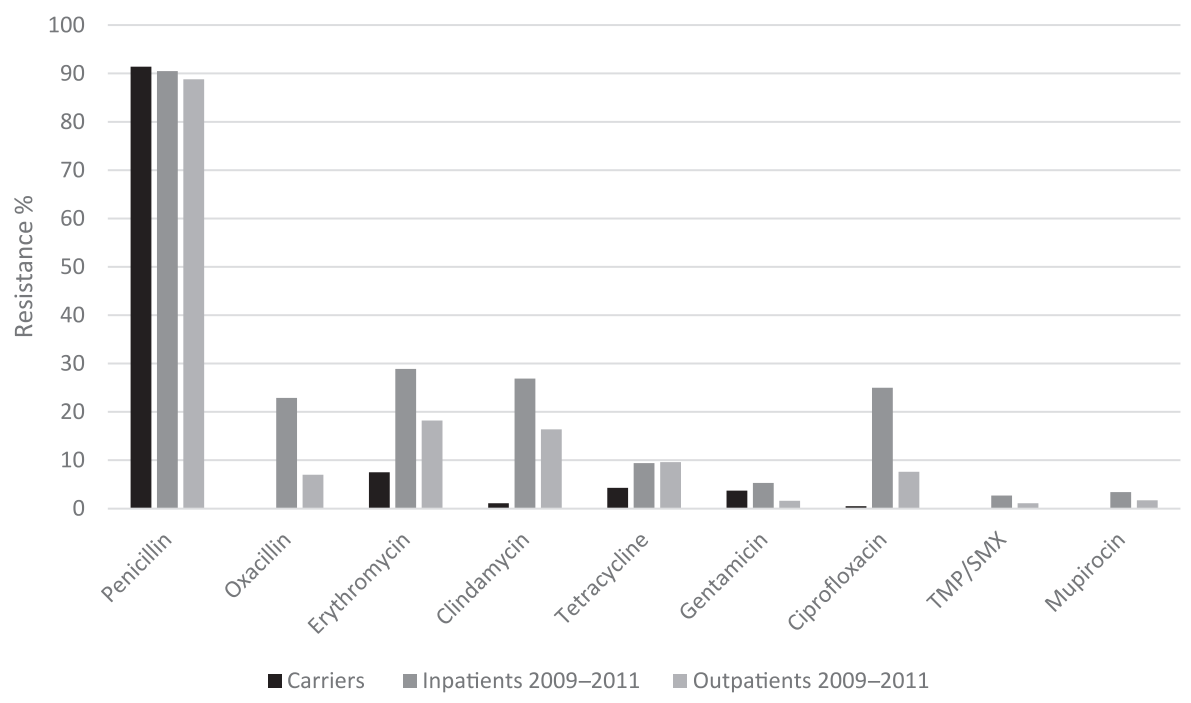

Figure 2. Antibiotic resistance rates of carried (this study, $n=187$ ) versus clinical (data obtained from the National Center for Epidemiology, $n=13,000-23,000$ each year) Staphylococcus aureus isolates in Hungary, in the period of 2009-2011. TMP/SMX: trimethoprim/sulfamethoxazole

over the last decade as seen in Figure 3, reaching already 9.8\% in 2015 (last available data). MRSA in inpatients was stable in the last 5 years. In this study, no MRSA carriers were found among the screened small children. On the other hand, in our previous survey among university students in Hungary, 2.3\% of carried S. aureus strains proved to be methicillin-resistant [11], but that rate is still much lower than that of clinical isolates. So based on our results, we can declare that unlike observed with the clinical isolates, CA-MRSA carriage in Hungary is very low yet.

MRSA prevalence varies widely in different geographical areas. Among 1,002 preschool children in India, $29 \%$ of the carried $S$. aureus isolates were MRSAs [9]. On the other hand, another research conducted in Italy demonstrated a prevalence of only $1.1 \%$ [10]. A recent Serbian paper reported 3.8\% MRSA carriage rate among 1,362 healthy adults in 2011-2013 [23]. Antibiotic sensitivity of carried S. aureus isolates in other European countries is more or less similar to that observed by us. For example, according to den Heijer et al. [16], erythromycin resistance varied between $1.6 \%$ in Sweden and 16.5\% in France; tetracycline resistance varied between $1.8 \%$ in Spain and $7.2 \%$ in Croatia. Little higher resistance rates were observed in Turkey compared with our results: erythromycin $16.7 \%$, clindamycin and tetracycline $8.3 \%$ [18]. However, there are countries with 


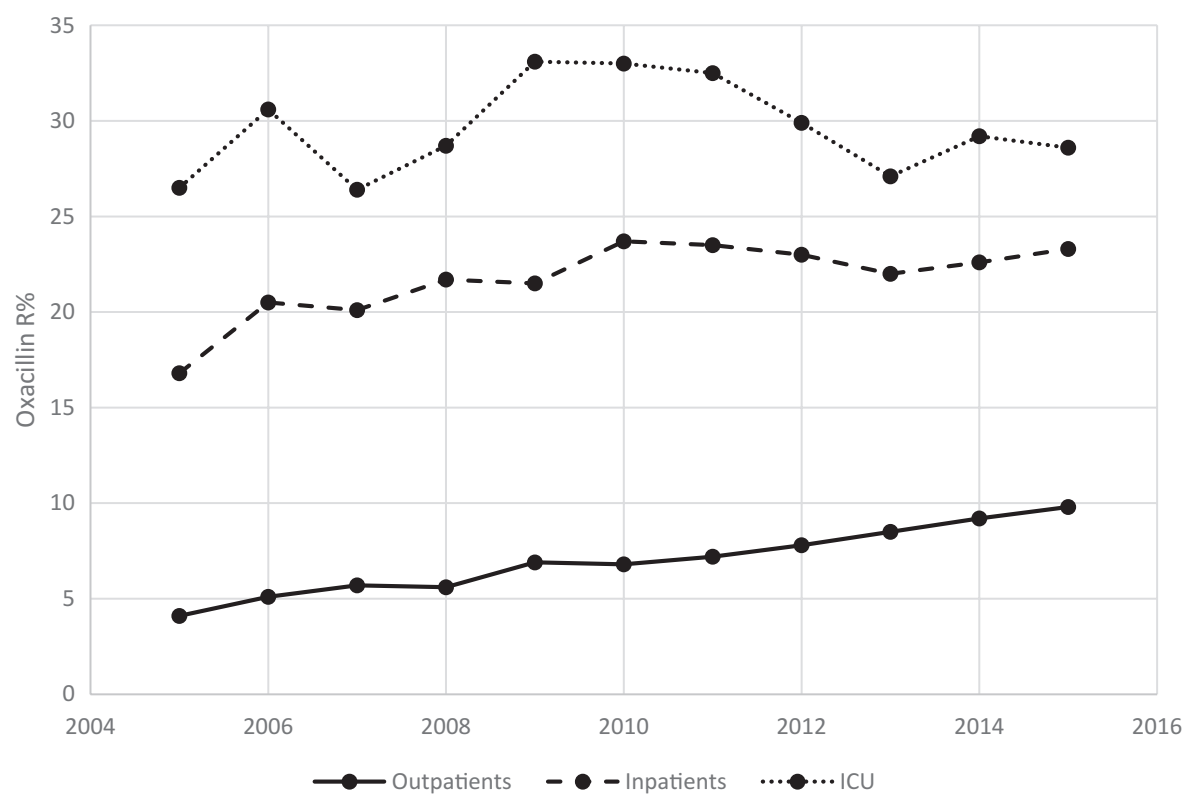

Figure 3. MRSA rates in Hungary among clinical isolates, between 2005 and 2015 (data obtained from the National Center for Epidemiology). ICU: intensive care unit

extremely high antibiotic resistance, such as India, where tetracycline resistance was found to be $41 \%$, gentamicin resistance $32 \%$, TMP/SMX resistance $28 \%$ [9], and ciprofloxacin resistance $23 \%$ [24].

The carried $S$. aureus isolates of this study were very diverse based on the PFGE patterns. This is in good concordance with other reports found in the literature. In a study by Ghasemzadeh-Moghaddam et al. [25], among 142 clinical and 110 CA-MSSA (community acquired methicillin-sensitive $S$. aureus) isolates, 98 different spa types were identified. Although clinical MRSA isolates tend to be rather clonal, in the case of carried MRSA isolates, the genetic diversity is high. In the European study of den Heijer et al. [16], 53 different spa types were recorded among the 91 carried MRSA strains.

\section{Acknowledgements}

Part of this paper was presented at the 24th ECCMID (P1605, Barcelona, 2014). This study was financially supported by the National Research, Development and Innovation Office/Hungarian Scientific Research Fund (grant number K108631). 


\section{Conflict of Interest}

The authors declare that they have no conflict of interest.

\section{References}

1. Kluytmans, J. A., Wertheim, H. F.: Nasal carriage of Staphylococcus aureus and prevention of nosocomial infections. Infection 33, 3-8 (2005).

2. Lee, G. M., Huang, S. S., Rifas-Shiman, S. L., Hinrichsen, V. L., Pelton, S. I., Kleinman, K., Hanage, W. P., Lipsitch, M., McAdam, A. J., Finkelstein, J. A.: Epidemiology and risk factors for Staphylococcus aureus colonization in children in the post-PCV7 era. BMC Infect Dis 9, 110 (2009).

3. Regev-Yochay, G., Dagan, R., Raz, M., Carmeli, Y., Shainberg, B., Derazne, E., Rahav, G., Rubinstein, E.: Association between carriage of Streptococcus pneumoniae and Staphylococcus aureus in children. JAMA 292, 716-720 (2004).

4. Ghasemzadeh-Moghaddam, H., Neela, V., van Wamel, W., Hamat, R. A., Shamsudin, M. N., Hussin, N. S., Aziz, M. N., Haspani, M. S., Johar, A., Thevarajah, S., Vos, M., van Belkum, A.: Nasal carriers are more likely to acquire exogenous Staphylococcus aureus strains than non-carriers. Clin Microbiol Infect 21, 998.e1-998.e7 (2015).

5. Sherertz, R. J., Bassetti, S., Bassetti-Wyss, B.: "Cloud" health-care workers. Emerg Infect Dis 7, 241-244 (2001).

6. Eichenwald, H. F., Kotsevalov, O., Fasso, L. A.: The "cloud baby": An example of bacterial-viral interaction. Am J Dis Child 100, 161-173 (1960).

7. Jourdain, S., Smeesters, P. R., Denis, O., Dramaix, M., Sputael, V., Malaviolle, X., Van Melderen, L., Vergison, A.: Differences in nasopharyngeal bacterial carriage in preschool children from different socio-economic origins. Clin Microbiol Infect 17, 907-914 (2011).

8. DeLeo, F. R., Otto, M., Kreiswirth, B. N., Chambers, H. F.: Community-associated methicillin-resistant Staphylococcus aureus. Lancet 375, 1557-1568 (2010).

9. Dey, S., Rosales-Klintz, S., Shouche, S., Pathak, J. P., Pathak, A.: Prevalence and risk factors for nasal carriage of Staphylococcus aureus in children attending anganwaries (preschools) in Ujjain, India. BMC Res Notes 6, 265 (2013).

10. Esposito, S., Terranova, L., Zampiero, A., Ierardi, V., Rios, W. P., Pelucchi, C., Principi, N.: Oropharyngeal and nasal Staphylococcus aureus carriage by healthy children. BMC Infect Dis 14, 723 (2014).

11. Laub, K., Kardos, S., Nagy, K., Dobay, O.: Detection of Staphylococcus aureus nasal carriage in healthy young adults from a Hungarian University. Acta Microbiol Immunol Hung 58, 75-84 (2011).

12. Paterson, G. K., Larsen, A. R., Robb, A., Edwards, G. E., Pennycott, T. W., Foster, G., Mot, D., Hermans, K., Baert, K., Peacock, S. J., Parkhill, J., Zadoks, R. N., Holmes, M. A.: The newly described mecA homologue, $m e c \mathrm{~A}_{\mathrm{LGA} 251}$, is present in methicillin-resistant Staphylococcus aureus isolates from a diverse range of host species. J Antimicrob Chemother 67, 2809-2813 (2012). 
13. The European Committee on Antimicrobial Susceptibility Testing. Breakpoint tables for interpretation of MICs and zone diameters. Version 6.0, 2016. Available at http://www. eucast.org/.

14. Szabó, J., Dombrádi, Z., Dobay, O., Orosi, P., Kónya, J., Nagy, K., Rozgonyi, F.: Phenotypic and genetic characterisation of methicillin-resistant Staphylococcus aureus strains isolated from the university hospitals of Debrecen. Eur J Clin Microbiol Infect Dis 28, 129-136 (2009).

15. Kumar, H., Palaha, R., Kaur, N., Ratnakar, W. S., Sodi, A., Kaur, M., Katiyar, R., Sharma, M., Kaur, C., Kumar, V.: Prevalence of multidrug-resistant, coagulase-positive Staphylococcus aureus in nasal carriage, food, wastewater and paper currency in Jalandhar city (northwestern), an Indian state of Punjab. Environ Monit Assess 187, 4134 (2015).

16. den Heijer, C. D., van Bijnen, E. M., Paget, W. J., Pringle, M., Goossens, H., Bruggeman, C. A., Schellevis, F. G., Stobberingh, E. E., Team, A. S.: Prevalence and resistance of commensal Staphylococcus aureus, including methicillin-resistant $S$. aureus, in nine European countries: A cross-sectional study. Lancet Infect Dis 13, 409-415 (2013).

17. Chatterjee, S. S., Ray, P., Aggarwal, A., Das, A., Sharma, M.: A community-based study on nasal carriage of Staphylococcus aureus. Indian J Med Res 130, 742-748 (2009).

18. Oguzkaya-Artan, M., Baykan, Z., Artan, C.: Nasal carriage of Staphylococcus aureus in healthy preschool children. Jpn J Infect Dis 61, 70-72 (2008).

19. Wertheim, H. F., Melles, D. C., Vos, M. C., van Leeuwen, W., van Belkum, A., Verbrugh, H. A., Nouwen, J. L.: The role of nasal carriage in Staphylococcus aureus infections. Lancet Infect Dis 5, 751-762 (2005).

20. Van Nguyen, K., Zhang, T., Thi Vu, B. N., Dao, T. T., Tran, T. K., Thi Nguyen, D. N., Thi Tran, H. K., Thi Nguyen, C. K., Fox, A., Horby, P., Wertheim, H.: Staphylococcus aureus nasopharyngeal carriage in rural and urban northern Vietnam. Trans R Soc Trop Med Hyg 108, 783-790 (2014).

21. Steiner, Z., Natan, O. B., Sukhotnik, I., Coran, A. G., Keren, G.: Does Staphylococcus aureus nasal carriage require eradication prior to elective ambulatory surgery in children? Pediatr Surg Int 30, 521-525 (2014).

22. National Bacteriological Surveillance Management Team: NBS Annual reports. National Center for Epidemiology, Budapest, Hungary, 2009-2011. Available at www.oek.hu.

23. Lepsanovic, Z., Jeremic, L. P., Lazic, S., Cirkovic, I.: High prevalence and resistance patterns of community-associated methicillin-resistant Staphylococcus aureus in the Pomoravlje region, Serbia. Acta Microbiol Immunol Hung 63, 83-92 (2016).

24. Pathak, A., Marothi, Y., Iyer, R. V., Singh, B., Sharma, M., Eriksson, B., Macaden, R., Lundborg, C. S.: Nasal carriage and antimicrobial susceptibility of Staphylococcus aureus in healthy preschool children in Ujjain, India. BMC Pediatr 10, 100 (2010).

25. Ghasemzadeh-Moghaddam, H., Ghaznavi-Rad, E., Sekawi, Z., Yun-Khoon, L., Aziz, M. N., Hamat, R. A., Melles, D. C., van Belkum, A., Shamsudin, M. N., Neela, V.: Methicillin-susceptible Staphylococcus aureus from clinical and community sources are genetically diverse. Int J Med Microbiol 301, 347-353 (2011). 